\title{
DETECTING AND PREVENTING ADVERSE DRUG INTERACTIONS: THE POTENTIAL CONTRIBUTION OF COMPUTERS IN PHARMACIES
}

\author{
Duane M. Kirking ${ }^{1.2}$, J. William Thomas ${ }^{2}$, Frank J. Ascione ${ }^{1}$ and Eddie L. Boyd \\ 'College of Pharmacy and 'Department of Medical Care Organization, School of Public Health, \\ The University of Michigan, 109 Observatory Street, Ann Arbor, MI 48109. U.S.A.
}

\begin{abstract}
For patients taking two or more medications concurrently, interactions among the drugs can cause undesirable effects or negate desired responses. In modern pharmacy practice, an important role of the pharmacist is to detect potentially harmful interactions and take appropriate action to prevent their occurrence. Pharmacy computer systems offer potential for improving pharmacists' effectiveness in the detection and followup of drug interactions

Based on a survey of southern Michigan pharmacists, relationships between computer use and pharmacists ${ }^{*}$ attitudes and activities in drug interaction monitoring were investigated. Respondents included users of two major computer systems as well as pharmacists who do not use computers. Results suggest that general statements cannot be made about the effect of computer use on drug interaction detection. Users of one of the two computer systems detected and followed up on interactions more frequently and were more likely to report improved knowledge of drug interactions than non-users. Frequencies of drug interaction detection and other related measures reported by users of the second computer system were similar to those for pharmacists not using computers. Computer system characteristics which might lead to these differences are discussed.
\end{abstract}

Key words -adverse drug reactions, computers, pharmacy, pharmacists, medication profile

A drug interaction occurs when an effect of one drug is altered by the prior or concurrent administration of another drug. Such interactions can produce undesirable effects on the patient or negate desired responses. For example, patients taking warfarin, an anticoagulant, who also receive the antiinflammatory drug phenylbutazone may experience serious bleed. ing episodes caused by an enhanced anticoagulant effect.

Not all of the interactions that occur are harmful to the patient. Furthermore, given the same combinations of drugs, some patients may experience interactions, while, because of differences in conditions and other factors which are not completely understood, others do not. Nevertheless, drug interactions constitute a significant medical care problem. Of the nearly 17,000 potentially interacting drug combinations which were identified in a recent study of outpatient pharmacy medication records, $6.3 \%$ were classified as likely to result in interactions in every exposed patient and to represent significant medical risks for those patients [1]. For another $26.7 \%$, interactions with potentially serious consequences were likely to occur in most exposed patients; and an additional $19.2 \%$ were classified as potentially significant depending upon individual patient conditions.

It has been estimated that as many as $27-33 \%$ of patients receiving more than one prescribed medication on an ambulatory basis are exposed to the possibility of interactions [1, 2]. Thus, these problems occur frequently, with serious consequences to the health of affected patients. and with resulting significant costs due to hospitalizations and physician care [3].
Prevention of drug interaction is difficult. Physicians are not always aware of all the drugs their patients are taking and may unwittingly prescribe drugs which lead to interactions, since patients often fail to give complete medication information to their physicians. In one survey of the elderly, for example, only $23 \%$ of respondents having two or more physicians reported discussing with one physician the medications prescribed by another [4].

Pharmacists, however, often are in a position to effectively monitor for drug interactions. Since $70-80 \%$ of patients receiving prescribed medications use a single pharmacy for all prescriptions $[5,6]$, pharmacists have the opportunity, as well as the training, to detect possible interactions and prevent their occurrence. For prescription medications, a pharmacist typically is the patient's final contact with the health care system before therapy is initiated. Also, patients requiring continuing medication therapy often visit their pharmacists for refills of prescriptions more frequently than they visit their physicians.

In this paper, we examine factors which influence how pharmacists perform this interaction monitoring task. Specifically, we focus on the likely contribution of computers, which are appearing with increasing frequency in community pharmacies, to pharmacists effectiveness in drug interaction monitoring.

\section{RESEARCH QUESTIONS}

For a pharmacist to monitor for drug interactions, an accurate chronological record of each of the patient's medications is needed, including, for each medication, its strength, the directions for its use, and 
the name of the prescriber. With this patient medication profile (PMP), the pharmacist can review the patient's active medication orders when a new drug is to be dispensed in order to search for other drugs with which the new medication might interact*. If a potential interaction is detected, the pharmacist can evaluate its significance and, if necessary, take measures to prevent its occurrence. In many instances, the pharmacist will need to contact the patient's physician about the problem to determine if an alternative treatment strategy is desirable.

Although the potential contribution of pharmacists in reducing the occurrence of adverse drug interactions is widely recognized, many pharmacists still do not actively maintain PMPs or systematically monitor for possible interactions. We suggest three primary factors as influencing this aspect of pharmacists' behavior: attitude toward the importance of drug interaction detection, level of technical knowledge of drug interactions, and the difficulty associated with the monitoring and detection process.

The clinical role of the pharmacist has been emphasized in recent years. While the importance of the pharmacist's role in preventing adverse drug interactions is recognized by both the pharmacy and medical professions $[7,8]$, this role is still not accepted by all practising pharmacists. It might be expected, then, that pharmacists who recognize interaction detection as an important aspect of their professional responsibilities would be more likely to perform the monitoring and detection function.

Although authoritative reference manuals on drug interactions are available $[9,10]$, it is unlikely that a pharmacist will have knowledge of all potentially interacting drug combinations that exist. Reports of newly identified interactions among both new and existing medications are published frequently. Pharmacists must not only be aware that a specific interaction can occur, they must also be able to judge the clinical significance of the interaction in specific situations.

The third factor believed to influence individual pharmacist's behavior in drug interaction detection is the difficulty and time experienced in maintaining PMP records, checking these records when prescriptions are filled, and following up with prescribers when appropriate. These activities must be performed in addition to pharmacists' more routine responsibilities such as preparing prescriptions to bc dispensed, obtaining payment from patients, and preparing third-party claims.

Computer systems are marketed to, and purchased by, pharmacists primarily for business and clerical purposes like inventory control, prescription container label printing and completion of insurance reimbursement claims. However, most systems also maintain patient medication profiles and many are programmed to search the PMPs for potential interactions. When a potential interaction is detected by the computer, the pharmacist is usually provided,

*While a complete patient medication profile would contain over-the-counter drugs, in practice they usually are not included because of difficulties associated with maintaining reliable information on patients' use of nonprescription medications. either on the monitor screen or through identification of the appropriate page in an interaction reference book. information such as the pharmacological action and clinical effects of the interaction and its frequency of occurrence.

Because of these capabilities, we hypothesize that pharmacists who use computers will be more likely to detect and followup on adverse drug interactions than those who do not. Specific questions addressed include:

How do drug interaction monitoring activities vary between pharmacists who use computers and those who do not?

Are computer users more or less likely than nonusers to act, when potential drug interactions are detected, by contacting the prescriber?

To what extent, if any, do computers influence pharmacists' knowledge of drug interactions, attitudes concerning the importance of interaction detection, and perceived difficulty in detecting clinically significant interactions?

\section{METHODOLOGY}

\section{Population description and sample selection}

The population for this study consisted of pharmacists who practice at least 20 hours per week in a community setting located in the 28 counties of southern Michigan. These counties were chosen because they contain over one-half of Michigan's pharmacies and represent a mix of urban and rural communities.

Pharmacists included in the survey were selected using a stratified cluster sampling approach in which the primary sampling units were pharmacies. Southern Michigan pharmacies were stratified into three groups based upon computer usage. Two groups of 66 pharmacies each were selected randomly from client lists obtained from the two large pharmacy computer vendors (labeled Computer A and B) serving Michigan pharmacies in the 28 county areas. After excluding users of Computers $A$ and $B, 80$ additional pharmacies were selected randomly from a Michigan Board of Pharmacy listing of all community pharmacies in southern Michigan. It was recognized that some pharmacies classified for sample selection purposes as non-users might actually be utilizing systems obtained from vendors other than $A$ or $B$, but it was expected that the number of such pharmacies would be relatively small. The assumption was verified by the fact that $77 \%$ of respondents in this group reported not having a computer. Each sample was selected to reflect the distribution of pharmacies in three geographic regions: the sevencounty Detroit metropolitan area, the seven counties in the middle part of the state, and the fourteen western counties.

Each pharmacy was contacted individually or through its chain headquarters, as appropriate, for the names and addresses of all pharmacists practicing more than 20 hours per week at that site. Two chains and four individual pharmacies ( 14 pharmacies in all) declined to participate, leaving a total of 198 cooperating in the study. From the participating pharmacies, 325 names were obtained, representing $100 \%$ of 
the eligible pharmacists at these sites. This list constituted the study sample.

\section{Survey procedure}

The results reported here are part of a larger survey of computer use in community pharmacy practice. The survey instrument included questions on the pharmacists' practice characteristics, and types and extent of professional activities performed each day, and attitudes toward specific professional responsibilities. Attitude questions were scored on 5-point Likert scales with appropriate anchors. For all questions, pharmacists were requested to respond in terms of their personal practices rather than the overall practices of their pharmacies.

As a result of a pilot test on a panel of 15 pharmacists, including non-users and users of Computers $\mathrm{A}$ and $\mathrm{B}$, several format and content changes to the questionnaire were made. The revised instrument was then distributed to pharmacists in the study sample using a procedure adapted from Dillman's Total Design Method which employs three mailings [11]. The initial and followup mailings contained a cover letter, a questionnaire and a stamped return envelope coded to allow identification of respondents for later mailings. Between these two mailings was a reminder mailing which consisted of a letter only.

\section{Analytical procedure}

The initial analysis, using two-tailed $t$-tests and $\chi^{2}$ tests, was designed to test general hypotheses on differences between computer users and non-users.

Analysis of variance with the Scheffe statistic was used for inter-group comparisons among non-users, users of Computer A and users of Computer B. The resulting analysis was conservative in that it controlled for the increased probability, when doing multiple comparisons, of falsely concluding that a difference between study groups exists ('experimentwise' error) [12]:

\section{Response rate and non-response analysis}

Of 325 surveys mailed, 218 were returned yielding a gross response rate of $67.1 \%$ and a net response rate of $65.7 \%$. Based on 213 usable responses, the respondent groups consisted of: Computer A, 81 responses; Computer B, 60 responses; all other computers, 16 responses; no computer, 56 responses.

Two analyses of non-response effects suggest that non-response bias was minimal. First, with additional data obtained from the Michigan Board of Pharmacy and the Michigan Pharmacists Association, respondents and non-respondents were compared using $\chi^{2}$ and $t$-tests, on six variables: year of licensure, type of practice (chain or independent), school from which graduated, qualification as internship preceptor, sex and urban/rural location. The respondent and non-

\section{* Pharmacist's individual workload was calculated as:}

$$
\text { Workload }=\text { PIPR }[1+(\text { HNPH } / \text { WH })]
$$

where PIPR is pharmacist's individual prescription rate in prescriptions per hour. HNPH is hours of nonprofessional help per day, and WH is pharmacist's workday in hours. respondent groups were not different with respect to the first four of these variables, but it was found that females and pharmacists from rural areas were more likely to respond. To test whether responses might be biased based on sex and region of respondent, fifty variables from the questionnaire were examined for relationships with these factors. These analyses failed to yield any important relationships, and no evidence of non-response bias was found.

The second analysis was based on the premise that if patterns of answers to specific questions are similar for individuals who responded to the initial, reminder and followup mailings, then the same patterns would hold for non-respondents, and non-response bias would be minimal [13]. Forty-two variables of primary interest were compared for respondents in the three groups above. In only two of the forty-two resulting analyses of variance was the $F$-valuc significant. Such a result would be expected by chance. Furthermore, for one variable the initial group was different from the other two groups, and for the other it was the reminder group.

\section{RESULTS}

The principal hypothesis of this study is that detection and followup of potential drug interactions will be improved by pharmacists' use of pharmacy computer systems. This hypothesis is investigated by comparing groups of computer users and non-users in terms of responses to questions concerning attitudes and activities related to drug interactions. To determine whether the observed differences between groups in drug interaction activities might be attributed to factors other than pharmacists' use of computers, user and non-user groups first were compared in terms of their personal and work-setting characteristics. In terms of respondents' sex, level of pharmacy education, years in practice, school attended, average number of prescriptions dispensed per hour and individual workload*, no significant differences were found between non-users and users of Computer $A$, users of Computer B, or the combined group of all computer users. Similarly, Computer A users did not differ from Computer B users on any of these attributes. Because of the small number of pharmacists using computer systems other than $A$ or $B$, these users were not compared as a sipparate group to the non-user and the Computer A and Computer B groups. However, they were included for comparisons between users and non-users.

In comparing characteristics of the pharmacies in which respondents practised, no significant differences were found among Computer A users, Computer B users and non-users in terms of the percentage of total sales represented by drugs and medical supplies. The pharmacies of Computer A users and Computer B users were not different in terms of percentage of prescriptions covered by thirdparty payors, total volume of prescriptions processed per hour and length of time the computer system had been installed. Pharmacies with computers appeared to process more prescriptions per hour than pharmacies not using computers, although, as noted above, prescription volume for individual pharmacists was not different among any of the groups. While Com- 
puter B users reported a higher proportion of thcir prescription volume covered by third-party payors $(66 \%)$ than did non-users $(59 \%)$, this difference does not appear to be of practical significance.

In terms of type of practice (chain versus independent), several differences were found among the groups. Fifty-eight percent of Computer A users were employed in chain stores compared to $34 \%$ of nonusers and only $9 \%$ of users of Computer B. Although the reasons for these differences are not known, they perhaps reflect different marketing strategies of computer system vendors. To determine whether type of practice was significant in explaining variability in any of the behavioral variables of interest, an analysis of variance was performed on each of the measures relating to drug interaction detection and followup activities. However, no significant effects were found, indicating that the differences, described below, in pharmacists' activities and attitudes concerning prevention of drug interactions appear not to be attributable to the pharmacists' personal characteristics or to characteristics of their practice settings, other than the use of a computer system.

To investigate whether pharmacists' use of computers helps explain the differences, a series of comparisons among non-users and users of computers $A$ and $B$ were performed. These results are presented in three areas. First, the likelihood of pharmacists detecting potential drug interactions is examined, along with pharmacists' attitudes toward this activity. Second, pharmacists' followup actions, in terms of contacting prescribers when potential drug interactions are detected, are described. The final section considers the impact of the computer and other sources of drug interaction information on pharmacists' knowledge of potential interactions.

\section{Activities and attitudes concerning drug interaction detection}

When asked how frequently they encountered potential drug interactions, computer users reported an average of 16.1 interactions detected per week compared to 8.7 for non-users. The difference between these two averages, although substantial, was not statistically significant due to a large difference in detection rates between users of computers $\mathbf{A}$ and $\mathbf{B}$. Users of Computer $A$ reported an average detection rate of 23.6 drug interactions per week, while the average rate for users of Computer B was only 7.3 per week. The rate for Computer $A$ users was significantly greater than that for non-users $(P<0.05)$ as well as that for Computer B users $(P<0.10)$; rates for non-users and Computer B users were not significantly different. These patterns do not appear to be attributable to differences in prescription volume since individual prescriptions dispensed per hour was not significantly different between computer users and non-users or between users of Computers $\mathrm{A}$ and $\mathrm{B}$.

Similar results were observed in respondents' views concerning the extent to which the detection of clinically significant drug interactions had increased during the past year. As shown in Table 1, computer users as a group reported a significantly greater increase than non-users. However, as in the question above, the difference was attributable to the larger

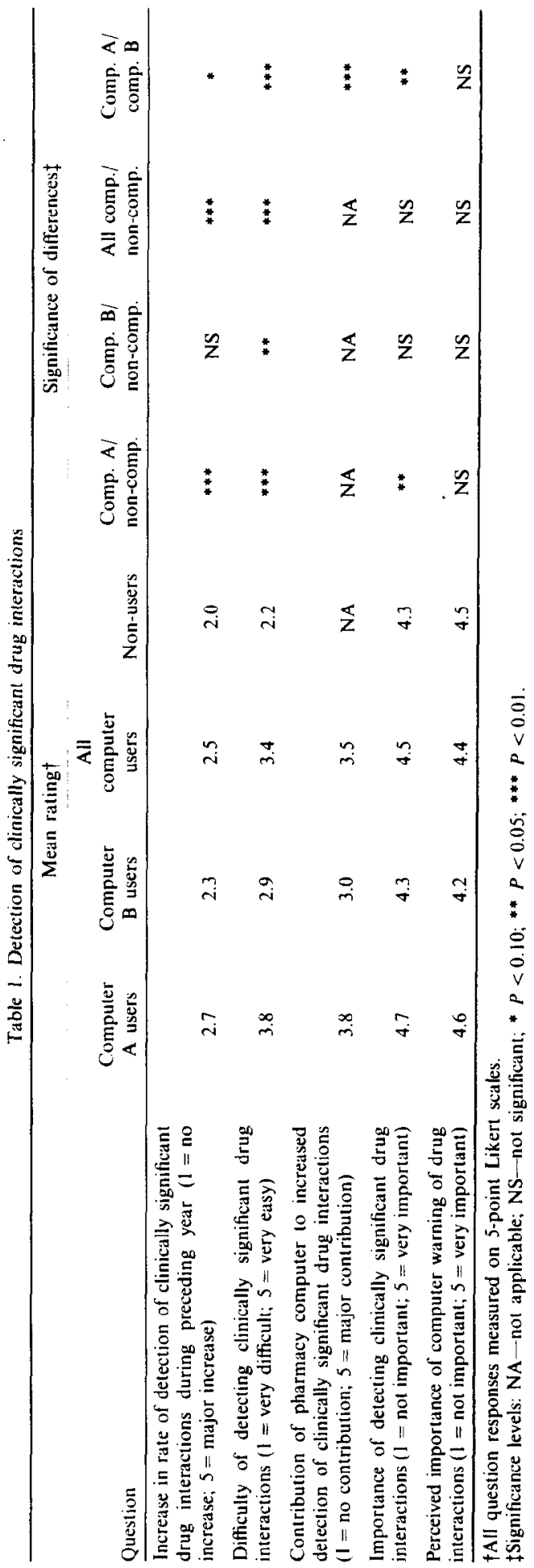


increases reported by Computer $A$ users. The extent of increase in drug interactions detected by Computer $B$ users was not significantly greater than that of non-users.

To determine pharmacists' views more directly regarding the usefulness of computers in detecting potential drug interactions, respondents were asked to indicate their perceptions of the degree of difficulty involved in detecting potential drug interactions and the extent to which the pharmacy computer (if applicable) contributed to the detection process. Both Computer $\mathbf{A}$ users and Computer $\mathbf{B}$ users reported significantly less difficulty than non-users in detecting potential interactions, and this same result was obtained when the group of all computer users was compared to non-users (see Table 1). However, consistent with previous findings, pharmacists using Computer A reported significantly less difficulty in detecting interactions and rated the contribution of the computer to the detection process significantly higher than did Computer B users.

Attitudes toward the importance of detecting drug interactions were assessed in two ways. First, pharmacists were asked directly about their opinions on the importance of detecting clinically significant drug interactions. As shown in Table 1, this function was considered important by all respondent groups, although it was viewed most positively by Computer $\mathrm{A}$ users. Second, the pharmacists were asked to rate the relative importance of 18 common applications of computer systems, including professional (e.g. warns of drug allergies), clerical (e.g. prints labels) and administrative (e.g. provides accounts receivable reports). All groups of computer users as well as non-users rated the 'warns of drug interactions' function as one of the most important. For non-users, this function was tied with 'provides tape-to-tape third party billing' as the most important function.

\section{Prescriber contacts regarding detected interactions}

Pharmacists commonly contact prescribing physicians by telephone for such purposes as clarification of prescription instructions or obtaining refill authorizations. Pharmacists also may contact prescribers regarding possible problems with prescriptions, such as when potential drug interactions or patient allergic reactions are detected. In such cases, the pharmacist may inform the physician of the problem and, as appropriate, discuss possible changes in therapy.

To investigate whether pharmacists who used computers were more or less likely to contact prescribers when drug interaction or allergy problems were detected, respondents were asked to indicate the percent of their total prescriber contacts which were for discussing a drug interaction or allergy problem. Respondents also provided an estimate of the total number of prescriber contacts they make per day. As a group, computer users when compared to non-users were found to have significantly more contacts per day with prescribers $(21.5$ vs 16.0 , respectively, $P<0.05$ ), and a significantly higher percentage of their contacts were related to interaction and allergy problems $(3.9 \%$ vs $2.8 \%$ respectively, $P<0.05)$. The higher percentage of such contacts by computer users appears to be due primarily to the Computer $A$ group, which reported an average of $4.4 \%$ of contacts for this purpose; the average percentage for Computer $\mathrm{B}$ users $(3.0 \%)$ was not significantly different from that of non-users.

\section{Computer's contribution to knowledge of drug inter- actions}

When asked whether their knowledge of drug interactions had changed during the past year, $81.5 \%$ of the Computer A users indicated that their knowledge in this area had increased. This was a higher percentage than that for non-users $(75.5 \%)$ and significantly higher $(P<0.05)$ than the $68.8 \%$ of Computer $\mathbf{B}$ users reporting an increase in knowledge.

Current information on drug interactions may be obtained by pharmacists from a variety of sources. To maintain or upgrade their knowledge in this area, pharmacists may consult books and other printed materials; also, they may communicate with pharmacists and other health professionals, attend continuing education seminars, and learn from experience with pharmacy computer systems. As shown in Table 2, when rating different sources of information in terms of relative contribution to respondents' knowledge of specific drug interactions, Computer A users rated the computer highest of the seven sources listed. This rating was significantly higher than that given the computer by users of Computer B, who suggested that the computer was a less important information source than journal articles, continuing education seminars, pharmacy and medical books and drug interaction references. Excluding the computer, which was not an applicable source of information for non-users, there were no significant differences in the ratings given the various sources by Computer $B$ users and by non-users.

Table 2. Contributions to pharmacists knowledge of drug interactions since pharmacy school graduation

\begin{tabular}{|c|c|c|c|c|c|c|c|c|}
\hline \multirow[b]{2}{*}{ Information source } & \multicolumn{4}{|c|}{ Relative importance of sourcet } & \multicolumn{4}{|c|}{ Significance of differences } \\
\hline & Comp. A & Comp. B & All comp. & Non-comp. & $\begin{array}{l}\text { Comp. A/ } \\
\text { non-comp. }\end{array}$ & $\begin{array}{l}\text { Comp. Bi } \\
\text { non-comp. }\end{array}$ & $\begin{array}{l}\text { All comp./ } \\
\text { non-comp. }\end{array}$ & $\begin{array}{l}\text { Comp. A } \\
\text { comp. B }\end{array}$ \\
\hline Pharmacy computer & 3.74 & 2.88 & 3.32 & NA & NA & NA & NA & $* *$ \\
\hline Drug interaction book(s) & 3.49 & 2.90 & 3.20 & 2.83 & $* * *$ & NS & $*$ & $* *$ \\
\hline Journal articles & 2.95 & 3.42 & 3.19 & 3.25 & NS & NS & NS & NS \\
\hline Continuing education seminar & 2.99 & 3.40 & 3.15 & 3.16 & NS & NS & NS & NS \\
\hline $\begin{array}{l}\text { Other pharmacy/medical books } \\
\text { Conversations with pharmacists }\end{array}$ & 2.77 & 3.20 & 2.92 & 3.09 & NS & NS & NS & NS \\
\hline and other health professionals & 2.80 & 2.73 & 2.75 & 2.59 & NS & NS & NS & NS \\
\hline Manufacturers' representatives & 2.25 & 2.76 & 2.44 & 2.40 & NS & NS & NS & $* *$ \\
\hline
\end{tabular}

+ Measured on 5-point scale ranging from $1=$ no contribution to $5=$ major contribution.

+ Significance levels: NA-not applicable: NS-not significant; $P<0.10 ;{ }^{* *} P<0.05$; $^{* * *} P<0.01$. 
Table 3. Availability in pharmacy of drug interaction references

\begin{tabular}{|c|c|c|c|c|c|c|c|c|}
\hline \multirow[b]{2}{*}{ References } & \multicolumn{4}{|c|}{$\%$ of respondents having references } & \multicolumn{4}{|c|}{ Significance of differences +} \\
\hline & Comp. A & Comp. B & All comp. & $\begin{array}{l}\text { Non- } \\
\text { comp. }\end{array}$ & $\begin{array}{l}\text { Comp. A } \\
\text { non-comp. }\end{array}$ & $\begin{array}{l}\text { Comp. B } \\
\text { non-comp. }\end{array}$ & $\begin{array}{l}\text { All comp. } \\
\text { non-comp. }\end{array}$ & $\begin{array}{c}\text { Comp. A } \\
\text { comp. B }\end{array}$ \\
\hline $\begin{array}{l}\text { One or both of the two } \\
\text { principal referencest }\end{array}$ & 92.6 & 76.7 & 86.1 & 63.6 & $* * *$ & NS & $* * *$ & $* * *$ \\
\hline Any reference & 97.5 & 91.7 & 94.9 & 81.8 & $* * *$ & NS & $* * *$ & $* * *$ \\
\hline
\end{tabular}

+American Pharmaceutical Association, Elaluation of Drug Interactions [9]: Hansten. Drug Interactions [10].

+Significance levels: NS-not significant: ${ }^{*} P<0.10:^{* *} P<0.05 ; * * * P<0.01$

Also, drug interaction books were rated significantly more important by Computer A users than by either non-users or users of Computer B. It is likely that ratings given for drug interaction references might be influenced by the types of information actually available in respondents' pharmacies. As shown in Table 3, at least one of the authoritative references on drug interactions $[9,10]$ was routinely available to the majority of respondents in each group. As might be expected given the ratings in Table 2, Computer A users were more likely than Computer B users and significantly more likely than non-users to have at least one of these references on hand. Although not statistically significant, the proportion of Computer B users with drug interaction information available in their pharmacies was greater than the proportion for non-users.

Computer users reported consulting these sources more frequently than non-users ( 5.3 vs 4.0 times per week, respectively), but this difference was not significant. A similar pattern was observed between users of Computer A and B (5.9 and 5.1 times/week, respectively). It is interesting to note that even for the group reporting the most frequent use of the books, Computer $\mathrm{A}$, the rate of referral was only once per day.

\section{DISCUSSION AND CONCLUSION}

The survey results suggest that the principal hypothesis of this study, that pharmacy computers promote increased detection and followup of drug interactions, cannot be accepted. One system, Computer A, appears to support and encourage pharmacists in this activity. The other computer system apparently has little effect.

The average frequency with which drug interactions were encountered by pharmacists who use computers was found not to be significantly different from the average frequency for pharmacists who do not use computers. Users of Computer A detected significantly more potential interactions than did non-users, but the number detected by users of Computer B was very nearly equal to that of nonusers. Likewise, the increase reported for the previous year in rate of detection of drug interactions was greater for computer users than for non-users, but this difference is attributable to the greater increase for users of Computer A. The change for Computer $B$ users was not significantly different from that of non-users.

The data show that for computer users the proportion of prescriber contacts which relate to drug problems (interactions, allergies) was significantly greater than the proportion for non-users. However, when non-users were compared to users of Computer
A and B separately, it was seen that the difference was significant only for users of Computer A. Again, the proportion for Computer B users was similar to that of non-users.

These patterns may be attributable to differences in pharmacists' perceived difficulty in detecting drug interactions or differences in pharmacists' knowledge of interactions. Although non-users reported a greater degree of difficulty in detecting potential drug interactions than did users of either Computer $A$ or B, the Computer A group was different from the B group in that Computer $A$ users reported significantly less difficulty, and they rated the contribution of the computer to their drug detection activities higher. The proportion of respondents who reported that their knowledge of drug interactions had increased during the previous year was greatest for Computer A users, and the proportion for Computer B users was smaller (although not significantly) than that of non-users. Non-users and Computer B users were similar to each other and different from Computer $\mathbf{A}$ users in their rankings of the importance of various sources of information on drug interactions. Users of Computer A ranked the computer as the most important source of information on drug interactions.

As noted above, non-users and users of Computers $A$ and $B$ do not differ in terms of personal characteristics such as education level and years of practice. While the proportion of Computer A users working in chain-store pharmacies is significantly greater than that for Computer B users and non-users, there is no reason to believe that pharmacists practising in chain store settings would be more likely than other pharmacists to detect potential drug interactions or to act upon those detected by contacting prescribers. Furthermore, analyses failed to show any significant relationship between practice setting and frequency of drug interaction detection, proportion of prescriber contacts relating to drug problems, or other major variables investigated. Therefore, it seems reasonable to hypothesize that differences observed in this study between Computer A users and other respondents are attributable to the computer system used.

While the scope of the present study does not allow us to identify those specific characteristics which make Computer A more effective than Computer B in supporting drug interaction detection and followup, we can speculate on some of the differences bctween the two systems. One difference which might influence monitoring behavior is the manner in which the pharmacist is referred to drug interaction references. When a potential interaction is detected, Computer $A$ refers the pharmacist directly to a specific 
page in one of the standard drug interaction books, which can be purchased from the computer vendor. Computer B, on the other hand, refers the user to the vendor's own manual. This book. in addition to summarizing the interaction, identifies relevant pages in the standard references if more information is desired. Although differences were not significant in all cases, compared to other respondents, users of Computer A were more likely to possess one or more of the standard reference books, tended to refer to these books with greater frequency, were more likely to report that their knowledge of drug interactions had increased during the previous year, and reportedly detected and followed up on more interactions.

Another design characteristic which might contribute to differences in pharmacists' detection activities is the manner in which the pharmacist is informed by the computer of potential interactions. For example, Computer A presents the pharmacist with information on both drugs involved in a potential interaction. Although Computer $\mathbf{B}$ indicates to the pharmacist that a potential drug interaction exists, it does not immediately identify which of the patient's current medications might interact with the newly-ordered drug. That information can be obtained if the pharmacist directs the computer to display a new screen on which the patient's medication profile is presented with the interacting drugs highlighted.

The number of potential drug interactions included in the computer database is another factor which might influence a system's effectiveness in supporting drug interaction detection. However, since the systems of both Computer $\mathrm{A}$ and $\mathrm{B}$ are based upon well-documented, comprehensive reference sources, there should be little difference between the two in the completeness of the data bases utilized.

Completeness may also be considered from a somewhat different perspective. If achieved at the cost of selectivity, completeness is not necessarily a desirable characteristic. For pharmacists in each of the respondent groups, the most commonly cited reason for choosing not to contact the prescriber when a potential drug interaction had been detected was the pharmacist's belief that harmful effects were unlikely to occur from that interaction. This finding suggests that a computer which identifies large numbers of interactions, including many which are generally not significant, may lead pharmacists to pay less attention to all warnings, including problems which are clinically more important. Thus, effectiveness of a pharmacy computer's drug interaction component might be greater if identification were limited to interactions which were more likely to be of clinical

*The selectivity issue is currently being investigated in a followup project to the research reported here. To test the importance of selectivity on pharmacists' use of drug interaction warning systems, a hospital pharmacy computer system has been modified so that detected interactions are flagged at one of three levels according to potential seriousness. and those with high potential for harm. as documented in the pharmacy and/or medical literature. cause the system to terminate medication order entry. Results of this project will be reported when available. significance. Since a detailed comparison of the specific drug interactions detected and reported by Computers A and B was beyond the scope of the current project, the degree to which differences in warning selectivity may have contributed to observed differences between users of $A$ and $B$ is not known.

The findings of this study highlight the need for further research. Detection and followup on potential drug interactions is an important aspect of pharmacists' professional responsibilities. Clearly, Computer $A$ is supportive of pharmacists efforts in this area while Computer B is not, and this difference appears to be due to specific design characteristics of the two systems. Additional research is needed to identify which characteristics are important in drug interaction detection and how they relate to pharmacists' attitudes and activities. Characteristics suggested by differences between Computers $\mathrm{A}$ and $\mathrm{B}$ in this study include: the specificity and completeness of reference sources to which pharmacists are directed when interactions are detected, the ease with which pharmacists can identify specific drugs involved when potential interactions are signaled, and the selectivity of the system as indicated by the proportion of signaled interactions which are of clinical significance* .

There are, of course, many factors not measured in this study which may influence pharmacists' ability or willingness to monitor for drug interactions. Attitudes of pharmacy management, receptiveness of prescribers toward suggestions made by pharmacists, and financial incentives to fill prescriptions may all be as important as computer system characteristics in promoting or discouraging drug interaction detection and followup. It would seem that efforts to refine computerized drug interaction monitoring should not be delayed until problems associated with these other factors are resolved, but rather should proceed concurrently. Such efiorts will not only improve the quality of drug interaction monitoring systems, but data from these systems may prove useful in promoting solutions to other problems preventing the reduction of harmful drug interactions.

\section{REFERENCES}

1. Shinn A. F., Shrewsbury R. P. and Anderson K. W. Development of a computerized drug interaction database (Medicom) for use in a patient specific environment. Drug Inform. J. 17, 205-210, 1983.

2. Mitchell G. W., Stanaszek W. F. and Nichols N. B Documenting drug-drug interactions in ambulatory patients. Am. J. Hosp. Pharm. 36, 653-657, 1979.

3. Silverman M., Lee P. R. and Lydecker M. N. Pills and the Public Purse: The Routes to National Drug Insurance. p. 31. University of California Press, Berkeley, 1981.

4. Ascione F. J., James M., Austin S. J. and Shimp L. A. Seniors and pharmacists: improving the dialogue. Am. Pharm. NS20, 270-272, 1980.

5. Laverty R. L. Patients loyal but need more Rx information. Drug Top. 128, 38-45, 1984

6. National Pharmacy Service Study. The Upjohn Co., Kalamazoo, Mich., 1979.

7. McLeod D. C. Contribution of clinical pharmacists to patient care. Am. J. Hosp. Pharm. 33, 904-911, 1976.

8. Lundberg G. D. The clinical pharmacist. J. Am. med. Ass. 249, $1193,1983$. 
9. Evaluation of drug interactions. American Pharmaceutical Association, Washington, D.C., 1976.

10. Hansten P. D. Drug Interactions. Lee \& Febiger. Philadelphia, 1979.

11. Dillman D. A. Mail and Telephone Surveys-The Total Design Method. Wiley. New York, 1978.
12. Neter J. and Wesserman W. Applied Linear Statistical Models. pp. 472-473, 477-479. Irwin. Homewood, Ill., 1974.

13. Churchill G. A. Marketing Research: Methodological Foundations, pp. 229-230. Dreyden Press, Hinsdale, Ill., 1976. 